rev.relac.int.estrateg.segur.5(1):13-31,2010

\title{
El LUgar de COREA del NORTE EN LA POLÍtiCa EXTERIOR DE LA FEDERACIÓN DE RUSIA UNA MIRADA A LA ÚLTIMA DÉCADA
}

\author{
Eduardo Carreño Lara*
}

\section{RESUMEN}

La relación entre la Federación de Rusia y la República Popular Democrática de Corea (Corea del Norte) ha fluctuado entre una cercana asociación estratégica y momentos de un virtual congelamiento de las relaciones. En este escenario, la administración de Vladimir Putin percibió en las recientes crisis nucleares norcoreanas una oportunidad para colocar nuevamente a su país en las "ligas mayores», pero esta vez apostando por un nuevo estilo de diplomacia que marcará a su sucesor Dmitri Medvedev.

Palabras clave: Rusia - Corea del norte - Corea del sur - armas nucleares - política exterior.

\footnotetext{
* Grados Académicos y Diplomas: Licenciado en Gobierno y Gestión Pública, Universidad de Chile. Magíster en Estudios Internacionales, Universidad de Chile. Diploma en Estudios Regionales (mención Asia Pacífico), Universidad de Santiago de Chile. Diploma en Procesos de Integración Regional, Universidad de Chile. Cargo Actual: Profesor-Investigador del Instituto de Estudios Internacionales de la Universidad de Chile. Dirección: Avenida Condell 249, Providencia, Santiago, Chile. ecarreno@uchile.cl
} 


\begin{abstract}
The relationship between the Russian Federation and the Democratic People's Republic of Korea (North Korea) has fluctuated between a nearly strategic association and moments of virtual freezing of their relations. In this scenario, Vladimir Putin's administration saw in the recent North Korean nuclear crisis an opportunity to put his country again in the «major leagues», but this time betting for a new style of diplomacy that will mark his successor Dmitri Medvedev.
\end{abstract}

Key words: Russia, North korea, South korea, nuclear weapons, foreign policy.

\title{
INTRODUCCIÓN
}

La política exterior de los Estados puede ser entendida, en términos generales, como la proyección de éstos hacia el sistema internacional, destacándose la relación con los demás Estados, organismos y actores internacionales, siguiéndose el marco de prioridades establecido por los líderes de cada país y que constituyen patrones conductuales a escoger entre diversos marcos de acción en pro de la consecución de los objetivos esenciales para un posicionamiento importante entre sus pares.

En este esfuerzo, los Estados se valen de diversos instrumentos de política exterior (políticos, económicos, ideológicos, culturales), siendo el determinante en la utilización de uno u otro la importancia asignada al asunto en cuestión; sin embargo, y desde un análisis a partir de la teoría realista de las relaciones internacionales, el fin último de todo Estado pasa por conservar y/o aumentar su capacidad de poder y grado de influencia en el sistema internacional. En efecto, el realismo supone que el concepto clave de «interés», definido como poder, es una categoría objetiva de validez universal, pero que no proporciona criterios para identificar intereses específicos, compararlos y establecer una jerarquía entre ellos para plantear opciones de política, ni para evaluar los costos y beneficios de ellas, provocando esto un aumento de la complejidad del sistema internacional debido a la proliferación de intereses contrapuestos (Tomassini 1987).

En este misma dirección, Carlos Eduardo Mena (1989) sostiene que el interés nacional se caracteriza principalmente por ser «una meta no operacional», es decir, al no haber una medida y al dificultarse así su comparación con otras políticas alternativas, todo juicio pierde objetividad, Ilevando esto a que el concepto sea utilizado de dos maneras distintas: primero, como un criterio para evaluar una situación determinada y adoptar el curso de acción que estime mejor el decisor; y segundo, como justificación para decisiones ya tomadas, adquiriendo esta última acepción mayor importancia con el aumento de la influencia de los medios de comunicación y de la opinión pública.

El interés político condicionará la conducta internacional de los Estados en un determinado período de la historia, pudiendo los objetivos que persiguen estos incluir un sinfín de ámbitos; no olvidándose que estas consideraciones son aplicables al concepto de «poder», el cual puede comprender cualquier 
cosa que establezca o mantenga el control del hombre sobre el hombre, desde la violencia física hasta el más sutil grado de influencia en una decisión (Morgenthau 1986). En este sentido, para George y Keohane (1980), el diseño, implementación y evaluación de la política exterior de los Estados supone una multiplicidad de valores e intereses particulares difíciles de armonizar, siendo necesario para el decisor aplicar el criterio de interés nacional a fin de reducir el problema de la complejidad axiológica y afrontar las incertidumbres que afectan la elección entre políticas alternativas.

La Federación de Rusia se encuentra precisamente en este escenario, donde luego del desmembramiento de la URSS ha visto cómo su posición de privilegio en el concierto internacional se ha perdido y la exclusión o la baja capacidad de influencia de esta en las discusiones políticas relevantes se ha hecho notar. Su papel en el Consejo de Seguridad si bien aun es considerable, principalmente por su capacidad de veto, se ha visto limitado por el debilitamiento que ha sufrido esta instancia a causa del accionar unilateral del otrora gran rival: Estados Unidos, como ocurrió, por ejemplo, en el caso de Kosovo en 1999 y en la invasión a Irak en 2003. En este sentido, la hipótesis de trabajo lleva a plantear que la búsqueda de un nuevo estatus en el sistema internacional por parte de la Federación de Rusia está estrechamente relacionada a un involucramiento activo por parte de Moscú en asuntos controversiales, y en este caso específico, en el noreste asiático, lo cual significa per se un accionar que se contrapone al de adversarios históricos como Washington y Tokio.

Rusia no está dispuesta a seguir siendo un actor secundario en el noreste asiático y en el concierto internacional, y el rol asumido en un tema tan álgido para la seguridad mundial, como la crisis en la península de Corea, es prueba de ello. La llegada al poder de Vladimir Putin en 1999 y, posteriormente, de Dmitri Medvedev en 2008 son instancias que pueden ser vistas como el inicio de un nuevo camino en la política exterior de la Federación de Rusia y que llevan a situarla, primero, como un actor más que relevante en el escenario regional; segundo, a un fortalecimiento de los lazos con socios estratégicos (Corea del Norte y Corea del Sur); y tercero, a un desplazamiento de esta zona de influencia de Estados Unidos y la República Popular China.

\section{RASGOS DE LA POLÍTICA EXTERIOR DE RUSIA}

Al igual que todo Estado, la Federación de Rusia en el desarrollo de su política exterior debe hacer frente a una serie de condicionantes internos que han ido moldeando su rol en el sistema internacional y que vienen a explicar su actual accionar. Corresponde a una política exterior que ha estado condicionada por la búsqueda de su seguridad mediante la dominación y un alto grado de influencia sobre las naciones vecinas, encontrándose una serie de factores domésticos que se constituyen como las principales fuentes de esta línea de acción: primero, optimización en la utilización de las fuentes de recursos naturales, implicando ello la consideración de una integración mayor de la Federación de Rusia al sistema económico de Oriente; segundo, la dificultad para construir una nación, aun cuando es cultural y étnicamente menos diversa que la ex Unión Soviética; 
tercero los problemas demográficos, debido a la combinación de una disminución en la tasa de natalidad y el incremento de la mortalidad, lo cual tendrá complicaciones en mediano y largo plazo en la definición de su política de seguridad y defensa; cuarto, la inestabilidad en la región del norte del Cáucaso y Chechenia, implicando esto una redefinición estratégica para enfrentar este escenario; quinto, el aumento de la percepción rusa de un ataque con armas de destrucción masiva a su territorio por parte de grupos terroristas, hecho que mantiene a Moscú en un trabajo conjunto con los EE.UU. para coordinar un desarme progresivo y frenar la proliferación de este tipo de armamentos; y sexto, la amenaza de nuevos fenómenos como el tráfico de drogas y la seguridad medioambiental, que han llevado a las autoridades rusas, por una parte, a reforzar lazos de cooperación con la región de Asia Central; y por otra, a convocar a la OTAN a un trabajo mancomunado en esta área (Troitskiy 2006).

En este escenario, a partir de 1991 se inicia un replanteamiento por parte de las nuevas autoridades rusas de la orientación que debería tomar su política exterior para enfrentar el nuevo contexto internacional, surgiendo propuestas que implicaban desde una reformulación total de esta y de sus rasgos históricos, hasta aquellas que abogaban por una mayor preocupación por asuntos internos. Blackwill, Braithwaite y Tanaka (1995: 27-28), al respecto sostienen que las alternativas consideradas para reformular la política exterior de la Federación de Rusia y sus nuevas prioridades podían ser clasificadas en cuatro grupos:

a. El grupo constituido por los más reformistas, los cuales apuntaron sin mayor dramatismo al establecimiento de una alianza estratégica junto a Estados Unidos y Europa Occidental.

b. Aquellos que veían a la Federación de Rusia como una potencia euroasiática con importantes intereses tanto en Occidente como en Oriente, enfoque que no veía con buenos ojos una excesiva dependencia rusa de EE.UU. y Europa Occidental. Para ellos, además, la República Popular China representaría una variable crítica e importante en el futuro de Moscú que debería ser considerada en la definición de los intereses nacionales.

c. Los sectores que abogaron por concentrarse en la periferia inmediata de la Federación de Rusia, argumentando que sus intereses nacionales dependían por sobre todo de lo que aconteciera en las repúblicas de la ex Unión Soviética.

d. Quienes creían que el reto más importante de la seguridad nacional rusa era de índole interna: mantener la propia integridad territorial.

El desmembramiento de la URSS implicó para la naciente Federación de Rusia establecer como principales objetivos el obtener el reconocimiento de la comunidad internacional, manteniendo, en lo posible, tanto el estatus de potencia internacional como su seguridad militar; quedando en esta primera etapa los asuntos económicos relegados, pero ocupando un lugar de privilegio en la agenda internacional rusa a partir de la llegada al Ministerio de Asuntos Exteriores de Andrei 
Kozyrev en 1992, quien optaría por dar a la política exterior una orientación manifiestamente pro occidental, intentando establecer nuevas alianzas en el ámbito político, económico y de la seguridad con quienes en un pasado bastante cercano fueron considerados rivales (Palacios y Arana 2002).

La llegada al poder de Vladimir Putin significó una verdadera reestructuración en la conducción de la política exterior rusa, donde el objetivo central pasó por recuperar el poder y la influencia tanto en la región euroasiática como en el sistema global. En sus primeros cuatro años de gobierno, Vladimir Putin se embarcó en el intento por sostener una política exterior pragmática, englobando las grandes amenazas a la civilización en el fenómeno del terrorismo e intentando hacer partícipe a Occidente en la lucha contra estas, lucha en la cual el fortalecimiento de la institucionalidad internacional es trascendental y para lo cual el ex Presidente ruso ya mostraba su disposición en su intervención en la Quincuagésima Octava Sesión de la Asamblea General de las Naciones Unidas el año 2003:

«Rusia está convencida de que el papel de las Naciones Unidas en los asuntos mundiales debe continuar siendo central. Y esto es por sobre todo relevante e importante para la resolución de situaciones de conflicto. Esta es nuestra opción y posición estratégica.»

El resurgimiento de la Federación de Rusia después de la gestión de Vladimir Putin es otra señal de que existen nuevas fuerzas en el mundo, rechazándose una visión unipolar de este en pro de crear un sistema político multipolar. Esa es la esencia que continúa con la «doctrina Medvedev», la cual se presenta como una propuesta de política exterior cuyo foco está en recomponer la quebrada sociedad rusa y en restablecer el lugar de Moscú en el mundo mediante una redefinición de los alcances de la seguridad internacional, del rol de la institucionalidad financiera mundial y de la presencia de EE.UU. en las zonas de influencia rusas, ya sea a través de un importante posicionamiento militar, o mediante el uso de sus enormes reservas de gas como instrumento de negociación y/o influencia (Sánchez y Carreño 2009).

A la luz de los acontecimientos históricos recientes, el análisis de la política exterior de Moscú durante este período revela la imposibilidad de recuperar el sitial de potencia internacional que se ocupó por muchos años, debido principalmente a un excesivo nacionalismo ruso que ha generado más de algún conflicto en regiones inestables como Chechenia y Georgia. Sin embargo, el análisis tampoco debe desconocer el impulso dado por el ex Presidente Putin a la política exterior, recuperando a través de su accionar algo de la pasada relevancia e influencia en el sistema internacional, pero esta vez de la mano no sólo de una aceptable capacidad militar, sino que también haciendo uso de una diplomacia proclive al fortalecimiento del derecho internacional, a la cooperación regional y mundial en temas álgidos y a la utilización de instrumentos internacionales efectivos más que efectistas en situaciones de compleja resolución. 


\section{¿POR QUÉ ES IMPORTANTE LA PENÍNSULA DE COREA EN LA POLÍTICA EXTERIOR DE LA FEDERACIÓN DE RUSIA?}

La relación entre Moscú y la península de Corea históricamente estuvo marcada por una complementariedad en sus intereses estratégicos con Corea del Norte, sin embargo, es posible identificar temas que han Ilevado a la Federación de Rusia a considerar de manera permanente dentro de sus prioridades la situación de la península en su conjunto. Estos pasan principalmente por los siguientes (Joo 1996):

a. Moscú y Seúl han estrechado sus lazos de interdependencia y mutua cooperación, pues la Federación de Rusia, mal que mal, necesita de la ayuda de Corea del Sur para potenciar su economía.

b. A diferencia de su relación con Japón, no hay ningún tema que sea fuente de conflicto entre Corea del Sur y la Federación de Rusia, excepto el descontento de Corea del Norte que ha sido marginada. Así, la política exterior de Moscú hacia Seúl es apenas susceptible a la presión interna de líderes conservadores y ultranacionalistas.

c. Las perspectivas de una proliferación nuclear en la península coreana y la resultante presencia de militares estadounidenses y chinos en la región están en los cálculos estratégicos de la Federación de Rusia, razón por la cual la prioridad más alta de Moscú está en restablecer una relación normal con Corea del Norte y persuadirla de no continuar con los incumplimientos de la normativa internacional.

Durante la permanencia en el poder de Gorbachev, las relaciones entre la ex URSS y la península de Corea comienzan a vivir cambios significativos, implicando esto hitos tan importantes como el establecimiento de relaciones diplomáticas con Corea del Sur y la consecuente decisión por parte de las autoridades de Pyongyang de rechazar este hecho y considerarlo una traición a los históricos vínculos que habían unido a los pueblos soviéticos y norcoreanos. Sin embargo, la decisión de Gorbachev de ampliar los vínculos en la península de Corea responden a cuestiones que tenían más implicancias internas que externas y que pasaban, primero, por la urgente necesidad de reducir costos en subsidios y ayuda militar, los cuales la economía soviética no podía soportar más; segundo, la visión negativa del tipo de comunismo de Corea del Norte entre los líderes reformistas en Moscú; y tercero, el atractivo de la cooperación económica con Corea del Sur, que era esperada para contribuir con la reestructuración y expansión de la economía soviética (Ellison 2001).

La llegada de Boris Yeltsin tampoco significaría un cambio en lo que respecta a la relación de la Federación de Rusia con la península de Corea y lo que habían sido las directrices fijadas durante la administración de Gorbachev, por el contrario, la administración de Yeltsin comenzó con un fuerte esfuerzo personal y de su gobierno por demostrar que la Federación de Rusia sería un activo promotor de la construcción de la democracia global, lo cual lo ayudaría a sustentar su propia 
reforma política y a construir lazos económicos para reestructurar su debilitada economía y fomentar el desarrollo.

Vladimir Putin, por su parte, ha buscado relacionarse con ambas Coreas en pos de la búsqueda de una mayor estabilidad en la región, principalmente considerando la amenaza que significa el desarrollo de armas de destrucción masiva al margen de toda normativa internacional por parte del régimen norcoreano. Para esto, el fomento de instancias multilaterales ha sido clave, buscado en estas concertar una sola visión a la hora de acordar soluciones más que transformarlas en un escenario de confrontación entre Estados Unidos y la Federación de Rusia en un tema de alta sensibilidad para todos los actores.

\section{RUSIA Y COREA DEL SUR}

El acercamiento entre Rusia y Corea del Sur, propiciado por los presidentes Gorbachev y Roh Taewoo, significó un giro trascendental en la orientación de la política exterior soviética, más aún con el establecimiento de relaciones diplomáticas en 1990, por cuanto este venía a romper en el noreste asiático con el clásico esquema de la Guerra Fría de las zonas influencia.

Boris Yeltsin fue aún más allá y propició en 1992, junto al mismo Roh Tae-woo, un tratado entre ambas partes tendiente a construir una relación sobre la base de ideales compartidos como la libertad, la democracia y el compromiso con la economía de mercado (Ko y Choi 2002). En efecto, corresponde a un acuerdo que representa cambios fundamentales en las percepciones y en las metas rusas en la península de Corea y en la región en su conjunto, por cuanto, primero, corresponde a un tratado que claramente refleja una nueva postura pro-surcoreana; segundo, el acuerdo transforma a la Federación de Rusia en la primera potencia internacional en establecer este tipo de mecanismo de concertación política con ambas Coreas, dándole una posición de privilegio en comparación con otras potencias como Japón, la República Popular China y EEUU; y tercero, la suscripción de este acuerdo implica el fin del clima de Guerra Fría en el noreste asiático (Ikjoong 2004).

El fin del gobierno de Yeltsin y el arribo de Vladimir Putin no cambió en demasía el escenario de cooperación que ha existido entre ambos países desde principios de los años noventa. Se ha seguido el camino establecido de la suscripción de acuerdos bilaterales, incluyéndose materias como comercio, garantías de inversión, pesca, prevención de doble tributación, cooperación en ámbito militar, cooperación en el tratamiento de material nuclear, asistencia mutua en investigación y desarrollo en el ámbito espacial, entre otras áreas (Ivashentsov 2006).

Un lugar destacado en la agenda de cooperación entre Seúl y Moscú ocupa la complementariedad en el sector energético, considerando especialmente la vulnerabilidad de Corea del Sur en la materia, que es el décimo consumidor mundial de energía y cuyo grado de autonomía es casi cero. La Federación de Rusia sabe de esta situación y al contar con grandes reservas de gas natural y petróleo 
REVISTA DE RELACIONES INTERNACIONALES, ESTRATEGIAYSEGURIDAD

ha apuntado a transformarse en un futuro cercano en el socio estratégico de Seúl en la materia mediante el desarrollo de una red que conecte el este de Siberia y el océano Pacífico y cuyo claro fin sería abastecer de petróleo a la península de Corea.

\section{RUSIA Y COREA DEL NORTE}

Tal como se mencionó anteriormente, la complementariedad entre Moscú y Pyongyang en intereses estratégicos ha estado presente desde finales de la Guerra de Corea, teniendo éstos su manifestación en diversos aspectos.

Evgeny Bazhanov (2003), en este sentido, sostiene que después de la Segunda Guerra Mundial tres importantes elementos sustentarían la política exterior soviética: la protección de la seguridad nacional, la extensión de la influencia del comunismo y el deseo de poder aumentar el territorio del imperio ruso; elementos que unidos a la rivalidad existente en esos años entre Moscú y Washington y materializada en la división de la península de Corea, Ilevarían a que las autoridades soviéticas no ahorraran esfuerzos en construir y reforzar un régimen leal en el norte de ésta.

Esta asociación estratégica se caracterizó por un alto nivel de dependencia de Corea del Norte en relación a la ex URSS, existiendo al respecto dos visiones explicativas de esta situación: por una parte, quienes sostienen que Moscú ha apostado siempre por un mantenimiento del status quo en la península de Corea; y por otra, quienes sostienen que si bien la URSS y luego la Federación de Rusia han considerado a Corea del Norte estratégicamente importante, han decidido fortalecer los lazos con Corea del Sur a fin de mejorar su situación económica, aun cuando esto signifique alejarse del régimen norcoreano (Woo 2005).

Desde el punto de vista ideológico, un análisis de la convergencia existente entre la ex URSS y el régimen norcoreano significa necesariamente considerar el rol jugado por la ideología Juche o autosuficiencia. Esta corresponde a la política llevada a cabo por Kim II Sung en 1955 y que encuentra su sustento en tres principios (Lee 2003): independencia interna y externa (Chaju); independencia económica (Charip), e independencia militar (Chawi); implicando esta doctrina ya un distanciamiento entre ambos regímenes comunistas, pero que vería en la adopción por parte de la URSS de una política menos confrontacional con Occidente ("Coexistencia Pacífica») un punto de inflexión importante, por cuanto corresponde a un alejamiento ideológico entre Moscú y Corea del Norte, y el acercamiento durante los años sesenta de este último con la República Popular China, aun cuando mantuviera su apoyo a los soviéticos en variados temas internacionales.

Por su parte, con la llegada de Khrushchev se inicia un período de «des-stalinización» de la política soviética, lo cual fue visto por Kim Il Sung como una amenaza a su propio régimen, acrecentándose esta visión por la negativa percepción generada en Pyongyang a raíz del accionar de Moscú en la crisis de los misiles en 1962 (International Crisis Group 2007). 
Con Gorbachev a la cabeza del gobierno soviético los lazos entre ambos regímenes inician un distanciamiento aún mayor, implicando incluso una reducción de la asistencia en materia militar (Cuadro $N^{\circ}$ 1). El acercamiento entre Moscú y Seúl, por su parte, se tradujo en un distanciamiento del régimen comunista norcoreano, generándose una profunda molestia en este último la cual Ilevaría a sus autoridades a tomar incluso una postura desafiante al nuevo enfoque de la política exterior de Gorbachev (Los Angeles Times; 2 de junio de 1990):

«Consideramos que el Presidente de la Unión Soviética, un aliado nuestro, es bastante capaz de analizar y juzgar lo que serían las consecuencias políticas que implican su encuentro con Roh Tae Woo, quien sólo busca hundir a Corea.»

Esta política será compartida desde 1991 por Boris Yeltsin y la nueva administración de la ahora Federación de Rusia, pues para este el complicado escenario económico y social que se enfrentaba implicaba dejar de lado cualquier prejuicio ideológico y establecer puentes concretos con el mundo occidental. En efecto, el interés central de la política exterior de Moscú hacia la península de Corea durante este periodo estuvo asociado a factores internos como los intereses económicos y el desarrollo, para lo cual una asociación con Pyongyang no significa mayores réditos, por el contrario, el régimen norcoreano era considerado anacrónico y sin futuro; visión que en cierto modo variaría en la segunda mitad de la década de los noventa al impulsarse una relación más «balanceada» con ambas Coreas (Joo 2003).

\section{Cuadro $\mathrm{N}^{\circ} 1$}

Transferencia de Sistemas de Armas de Rusia a Corea del Norte (1991-2000)

\begin{tabular}{|c|c|c|c|c|c}
\hline $\begin{array}{c}\text { TIPO DE } \\
\text { ARMAMENTO }\end{array}$ & $\begin{array}{c}\mathbf{N}^{\circ} \\
\text { ORDENADO }\end{array}$ & DESCRIPCIÓN & $\begin{array}{c}\text { AÑO DE } \\
\text { ORDEN/LICENCIA }\end{array}$ & $\begin{array}{c}\text { AÑO DE } \\
\text { DESPACHO }\end{array}$ & $\begin{array}{c}\mathbf{N}^{\circ} \\
\text { DESPACHADO/ } \\
\text { PRODUCIDO }\end{array}$ \\
\hline $\begin{array}{c}\text { MR-104/Drum } \\
\text { Tilt }\end{array}$ & $(6)$ & $\begin{array}{c}\text { Radar de } \\
\text { Control de } \\
\text { Fuego }\end{array}$ & $(1979)$ & $1992-1996$ & $(6)$ \\
\hline $\begin{array}{c}\text { P-15U/SS-N- } \\
\text { 2B Styx }\end{array}$ & $(35)$ & $\begin{array}{c}\text { Misiles } \\
\text { Antibuques }\end{array}$ & $(1979)$ & $1992-1996$ & $(35)$ \\
\hline $\begin{array}{c}\text { Rangout/Squar } \\
\text { e Tie }\end{array}$ & $(4)$ & Radares & $(1979)$ & $1992-1996$ & $(4)$ \\
\hline Mi-26/Halo & $(4)$ & Helicópteros & $(1994)$ & $1995-1996$ & $(4)$ \\
\hline $\begin{array}{c}\text { BTR-80A } \\
\text { Vehículos de } \\
\text { Combate de } \\
\text { Infantería }\end{array}$ & $\begin{array}{c}\text { Misiles } \\
\text { Antitanques }\end{array}$ & $(2000)$ & $2000-2001$ & 32 \\
\hline $\begin{array}{c}\text { SM14Mgar } \\
\text { (Licencia) }\end{array}$ & $(4000)$ & 1975 & $1992-1995$ & $(4000)$ \\
\hline
\end{tabular}


REVISTA DE RELACIONES INTERNACIONALES, ESTRATEGIAY YEGURIDAD

\begin{tabular}{|c|c|c|c|c|c|}
$\begin{array}{c}\text { Strela-2/SA-7 } \\
\text { Grail (Licencia) }\end{array}$ & $(500)$ & $\begin{array}{c}\text { Misiles tierra- } \\
\text { aire portátiles }\end{array}$ & (1985) & $1992-1993$ & (500) \\
\hline $\begin{array}{c}\text { 9M111/AT -4 } \\
\text { Spigot } \\
\text { (Licencia) }\end{array}$ & -- & $\begin{array}{c}\text { Misiles } \\
\text { Antitanques }\end{array}$ & $(1987)$ & $1992-2009$ & $(3250)$ \\
\hline $\begin{array}{c}\text { Igla-1/SA-16 } \\
\text { Gimlet } \\
\text { (Licencia) }\end{array}$ & -- & $\begin{array}{c}\text { Misiles tierra- } \\
\text { aire portátiles }\end{array}$ & $\mathbf{1 9 8 9 )}$ & $1992-2009$ & $\mathbf{1 1 5 0 0 )}$ \\
\hline
\end{tabular}

Fuente: Elaboración propia con base en los datos obtenidos de Stockholm International Peace Research Institute (SIPRI), www.sipri.org/database

Bajo la administración de Vladimir Putin se inicia un refortalecimiento de los vínculos entre Moscú y el régimen de Pyongyang, produciéndose los mayores avances a inicios del siglo XXI, destacando la reactivación del diálogo político-diplomático entre ambos países en el año 2000. Por esos días, además, se concretaría la visita del Ministro de Relaciones Exteriores Igor Ivanov a Corea del Norte y la firma durante la misma de un nuevo Tratado de Amistad y Cooperación que reemplazaría al suscrito por allá en los años sesenta.

Este hecho es de suma importancia, por cuanto implica la suscripción de un tratado que no incluía una cláusula automática de intervención militar como el tratado de 1961, sino que estipulaba el establecimiento de mecanismos de consulta bilateral en materias de seguridad si una de las partes se veía amenazada. Además, la importancia de este nuevo tratado se ve reflejada en la intención de ambas partes de obligarse a no concluir ningún tratado o acuerdo con un tercer país ni participar de su acción si estos están contra la soberanía, independencia e integridad territorial de cualquiera de los contratantes. Por otro lado, la visita realizada por el Presidente Putin a Pyongyang, previa a su reunión en el G8 en julio de 2000, representó para las autoridades norcoreanas la recomposición definitiva de lazos, pero a la vez significaba un reposicionamiento de Moscú en la región, deseo que estaba presente desde un comienzo y que encontró en este gesto su materialización (Takeda 2006). En efecto, este tipo de acciones se enmarca, en declaraciones del propio ministro Ivanov, dentro de las prioridades regionales establecidas por la Federación de Rusia y que habla de buscar participar en la discusión de temas relevantes e impulsar una integración económica y en el ámbito de la seguridad en pro de asegurar sus propios intereses en las zonas fronterizas (Buszynski 2000).

\section{LA CRISIS NUCLEAR EN LA PENÍNSULA DE COREA Y LAS IMPLICANCIAS PARA RUSIA}

El desarrollo del programa nuclear de Corea del Norte está estrechamente ligado a la cooperación y asistencia militar histórica dada por Moscú, en especial, a partir de 1956, fecha en que la ex URSS fortalece la promoción del desarrollo tecnológico en su zona de influencia como respuesta 
al programa similar impulsado por Estados Unidos, y que se materializó en el entrenamiento de personal norcoreano en tierras soviéticas y en el desarrollo de reactores nucleares.

Los años posteriores significaron un escalamiento de la tensión en la zona, implicando ello un reposicionamiento del tema en la agenda de seguridad internacional. En 1993, Corea del Norte, desconociendo el acuerdo suscrito con la Agencia Internacional de Energía Atómica (AIEA) el año anterior, rechaza la inspección de observadores internacionales, quienes habían advertido sobre las sospechas existentes de la posesión no declarada de plutonio en la zona. Pyongyang endurece su postura y amenaza con el retiro del Tratado de No Proliferación Nuclear, debiendo Estados Unidos buscar un acercamiento con el régimen comunista cuyo resultado fue un inesperado acuerdo en 1994 que implicaba el compromiso de Corea del Norte de abandonar su programa nuclear a cambio de la construcción de dos reactores nucleares de agua ligera para la generación de electricidad, así como también la entrega de medio millón de toneladas de petróleo al año hasta la finalización del primer reactor (Villanueva; 2006).

Durante el transcurso del año 2002, nuevamente contraviniendo las normativas internacionales, Corea del Norte reanuda su programa nuclear y declara abiertamente su intención de continuar con el enriquecimiento de uranio. EE.UU. manifiesta su profundo rechazo a este hecho, debido a sus fundadas sospechas del intento de régimen de Pyongyang de orientar el mencionado programa al desarrollo de armas de destrucción masiva.

\section{Cuadro $\mathrm{N}^{\circ} 2$}

\section{Estimación del Arsenal de Misiles Balísticos de Corea del Norte}

\begin{tabular}{|c|c|c|c|c|c|}
\hline Tipo & $\begin{array}{c}\text { Alcance } \\
\text { (Kilómetros) }\end{array}$ & $\begin{array}{c}\text { Carga } \\
\text { (Kilogramos) }\end{array}$ & Ojiva & $\begin{array}{c}\text { Probables } \\
\text { objetivos }\end{array}$ & Estado \\
\hline $\begin{array}{c}\text { KN-02 } \\
\text { (modificación } \\
\text { SS-21 } \\
\text { Scarab) }\end{array}$ & $100-120$ & 250 & Convencional & $\begin{array}{l}\text { Objetivos } \\
\text { tácticos } \\
\text { en Corea } \\
\text { del Sur }\end{array}$ & En prueba \\
\hline $\begin{array}{l}\text { Hwasong-5 } \\
\text { (Scud-B) }\end{array}$ & 300 & $987-989$ & $\begin{array}{c}\text { Convencional; } \\
\text { posiblemente } \\
\text { nuclear, } \\
\text { biológica o } \\
\text { química }\end{array}$ & $\begin{array}{c}\text { Corea del } \\
\text { Sur }\end{array}$ & $\begin{array}{c}\text { Desarrollado } \\
\text { Exportado }\end{array}$ \\
\hline $\begin{array}{c}\text { Hwasong-6 } \\
\text { (Scud-C) }\end{array}$ & $\begin{array}{c}500 \\
\text { (estimado) }\end{array}$ & 770 & $\begin{array}{c}\text { Convencional; } \\
\text { posiblemente } \\
\text { nuclear, } \\
\text { biológica o } \\
\text { química }\end{array}$ & $\begin{array}{c}\text { Corea del } \\
\text { Sur }\end{array}$ & $\begin{array}{c}\text { Desarrollado } \\
\text { Exportado }\end{array}$ \\
\hline
\end{tabular}




\begin{tabular}{|c|c|c|c|c|c|}
\hline Scud-D & $\begin{array}{c}700 \\
\text { (estimado) }\end{array}$ & 500 & $\begin{array}{l}\text { Convencional; } \\
\text { sin } \\
\text { información } \\
\text { sobre otros } \\
\text { tipos }\end{array}$ & $\begin{array}{l}\text { Corea del } \\
\text { Sur }\end{array}$ & $\begin{array}{c}\text { Desarrollado } \\
\text { Exportado }\end{array}$ \\
\hline Nodong & $\begin{array}{c}1.000 \\
\text { (estimado) }\end{array}$ & 700 & $\begin{array}{l}\text { Convencional; } \\
\text { posiblemente } \\
\text { nuclear, } \\
\text { biológica o } \\
\text { química }\end{array}$ & Japón & $\begin{array}{c}\text { Desarrollado } \\
\text { Exportado }\end{array}$ \\
\hline $\begin{array}{c}\text { Taepodong- } \\
\text { X (R-27/SS- } \\
\text { N-6) }\end{array}$ & $\begin{array}{c}2.500-4.000 \\
\text { (estimado) }\end{array}$ & $\begin{array}{c}\text { Sin } \\
\text { información }\end{array}$ & $\begin{array}{c}\text { Convencional; } \\
\text { posiblemente } \\
\text { nuclear, } \\
\text { biológica o } \\
\text { química }\end{array}$ & $\begin{array}{l}\text { Japón, } \\
\text { Okinawa, } \\
\text { Guam }\end{array}$ & $\begin{array}{c}\text { ¿Desarrollado? } \\
\text { ¿Exportado? }\end{array}$ \\
\hline $\begin{array}{c}\text { Paektusan-1 } \\
\text { (Taepodong- } \\
1 ; \text { dos } \\
\text { etapas) }\end{array}$ & $\begin{array}{c}2.200 \\
\text { (estimado) }\end{array}$ & $\begin{array}{c}\text { Sin } \\
\text { información }\end{array}$ & $\begin{array}{l}\text { Convencional; } \\
\text { posiblemente } \\
\text { nuclear, } \\
\text { biológica o } \\
\text { química }\end{array}$ & $\begin{array}{l}\text { Japón, } \\
\text { Okinawa, } \\
\text { Guam }\end{array}$ & $\begin{array}{c}\text { En prueba } \\
\text { ¿Desarrollado? } \\
\text { ¿Exportado? }\end{array}$ \\
\hline $\begin{array}{c}\text { Taepodong- } \\
2\end{array}$ & $\begin{array}{l}\text { ¿5.000- } \\
6.000 ?\end{array}$ & $\begin{array}{c}\text { Sin } \\
\text { información }\end{array}$ & $\begin{array}{c}\text { Convencional; } \\
\text { posiblemente } \\
\text { nuclear, } \\
\text { biológica o }\end{array}$ & EE.UU. & $\begin{array}{c}\text { I\&D } \\
\text { Prototipo } \\
\text { En prueba }\end{array}$ \\
\hline
\end{tabular}

Fuente: Adaptación de East Asia Nonproliferation Program (EANP); 1996

Para la Federación de Rusia el establecimiento de un diálogo multilateral ha sido el camino escogido para solucionar la crisis nuclear de la península de Corea, desarrollando el ex Presidente Putin y su gobierno una estrategia tendiente a presentarse a sí mismos como un país poderoso y respetado en la región, pero esta vez en virtud de la utilización de un soft power y un smart power ${ }^{1}$, directrices

\footnotetext{
${ }^{1}$ El concepto de «soft power» corresponde a la habilidad para obtener lo que se quiere a través de la persuasión y la atracción, más que utilizando la coerción; mientras que «smart power» implica la capacidad de un actor 
diplomáticas consideradas por Moscú en este escenario mucho más efectivas que la imposición de un sinnúmero de sanciones. Al respecto, Samuel Kim (2007) sostiene que tras la muerte de Kim II Sung, Corea del Norte ha estado sujeta a las mismas presiones externas y dinámicas propias de un mundo interdependiente e interactivo; por lo tanto, la proyección internacional del régimen norcoreano y que define su estatus es el resultado de una trayectoria que Pyongyang ha elegido o ha sido forzada a tomar dado sus intereses nacionales: la sobrevivencia del régimen, la obtención de ayuda económica externa, restablecimiento del balance de poder en la península, entre otros.

En el contexto del Diálogo a Seis a Bandas que integran ambas Coreas, Estados Unidos, la Federación de Rusia, Japón y la República Popular China, se ha propiciado el desmantelamiento del programa nuclear de Corea del Norte y la canalización de las demandas del régimen de Pyongyang a fin de obtener la asistencia de la comunidad internacional como moneda de cambio al primer punto. En este sentido, la Federación de Rusia ha sido particularmente crítica del accionar de EE.UU. y Japón², mostrando, a diferencia de los demás países involucrados en las conversaciones, una mayor compasión hacia la posición de Pyongyang y un deseo de que el programa nuclear norcoreano se ajuste a la normativa internacional y adquiera definitivamente una orientación pacífica (Kimura 2005).

Se ha buscado una solución política, agotándose primero todos los medios diplomáticos disponibles para frenar cualquier intento de Corea del Norte de utilizar su capacidad nuclear con fines militares; asumiendo Moscú para ello diferentes roles durante el proceso de paz (Joo 2004):

a. Garante de la política exterior de Corea del Norte: Los encargados de la diplomacia de la Federación de Rusia sostienen que una vez restablecida la amistad y la confianza con el régimen de Pyongyang, es posible preservar tanto la seguridad de la región como de la península de Corea, razón por la cual el ex Presidente Putin se transformó en un garante del comportamiento internacional de Kim Jong Il y de su gobierno.

b. Mediador: la Federación de Rusia mantiene permanentes contactos con Corea del Norte a través de la Embajada de esta en Moscú, de sus enviados especiales para la causa y de otros variados canales, a fin de hacer posible el diálogo directo de Kim Jong Il y su gobierno con Seúl, pero manteniendo siempre un rol indirecto, sin inmiscuirse mayormente en la toma de decisiones.

de combinar diversos instrumentos de política exterior (políticos, económicos, militares, culturales) en aras de alcanzar objetivos de manera eficaz y eficiente. Véase: Wilson 2008

2 Para las autoridades rusas, que Japón presentara en esta instancia su histórica protesta a Corea del Norte por los secuestros de sus connacionales, es un error, por cuanto la desnuclearización de la península Coreana es el eje central de la discusión y el hecho de que una de las partes trate de reorientar el objetivo, distorsiona el clima de negociación; más aún considerando el importante rol de Japón en la discusión, pues es este quien posee uno de los instrumentos claves para disuadir a Pyongyang de desestabilizar la región: asistencia económica para recuperar el país. 
c. Crítico del rol de EE.UU.: las discrepancias entre la Federación de Rusia y Estados Unidos han pasado permanentemente por cómo abordar el tema de la crisis nuclear, en especial el cartel dado por parte de Washington al régimen de Kim Jong Il de sostenedor del terrorismo. Moscú ha recomendado a EE.UU. a lo largo de todo el proceso de paz el levantamiento de las sanciones impuestas y la colaboración en la asistencia en materia energética a Corea del Norte como la mejor herramienta para frenar cualquier amenaza a la seguridad regional.

Internacionalmente, Vladimir Putin fue capaz de dar un nuevo rostro a la Federación de Rusia, asumió sus desventajas como país y concluyó que el restablecimiento del equilibrio estratégico pasa hoy por hoy por un acercamiento a nivel multilateral a Estados Unidos y a las demás potencias del noreste asiático. En este escenario, el apoyo de la Federación de Rusia a las propuestas de desnuclearización y no proliferación de armas de destrucción masiva en la península de Corea tiene detrás precisamente el tratar de equilibrar la capacidad de influencia en la región de Washington, Beijing y Tokio, pero esta vez ejecutando una política exterior que no sólo se sustenta en su importante poder militar, sino que también ha sabido combinar el histórico desarrollo de este con una alta capacidad de negociación y persuasión diplomática.

\section{La Administración Medvedev: ¿ icontinuismo o rupturismo en la política exterior rusa hacia el régimen norcoreano?}

La política exterior de la Federación de Rusia hacia Corea del Norte durante los primeros años de gobierno del Presidente Medvedev ha estado marcada por el recrudecimiento de la tensión en la región, producto de las nuevas pruebas con misiles balísticos de corto alcance impulsadas por Pyongyang, contraviniéndose así las disposiciones establecidas en la Resolución 1718 (2006) del Consejo de Seguridad de las Naciones Unidas y que prohíbe este tipo de acciones.

El objetivo del Presidente Medvedev al inicio de su Administración pasaba por aprovechar el interesante lugar alcanzado por la Federación de Rusia en el marco del Diálogo a Seis a Bandas y potenciar su rol como mediador entre ambas coreas en pro de concretar una serie de proyectos de cooperación energética y de interconexión ferroviaria que significarían en el mediano y largo plazo, por una parte, importantes beneficios políticos, estratégicos y económicos para Moscú; y por otra, el establecimiento de un statu quo en la relación bilateral entre ambas coreas.

En esta dirección, las reuniones sostenidas entre el Presidente Medvedev y el Presidente surcoreano Lee Myung-bak, en octubre de 2008, apuntaron a potenciar diferentes ámbitos de la relación bilateral entre ambos países, destacándose la designación del año 2010 como el «Año de Corea y el Año de Rusia», instancia que permitirá profundizar el entendimiento de los pueblos de ambos países mediante la realización de una serie de actividades culturales que se darán en el marco del vigésimo aniversario del establecimiento de relaciones diplomáticas entre Moscú y Seúl³. Además, en el

\footnotetext{
${ }^{3}$ Es importante destacar también el fortalecimiento de la relación bilateral entre Corea del Sur y la Federación de Rusia en el ámbito militar, siendo prueba de ello el interés mostrado por Seúl el año 2008 por adquirir a la 
plano de la relación con Pyongyang, la diplomacia rusa continuó teniendo gestos de simpatía para con el régimen de Kim Jong II, destacando los buenos deseos expuestos por la Administración Medvedev en el saludo enviado en abril de 2009 a las autoridades norcoreanas por motivo del $97^{\circ}$ aniversario del nacimiento de Presidente Kim II Sung:

«Estoy convencido de que en el futuro también los esfuerzos comunes de nuestros dos países por seguir ampliando y fortaleciendo la cooperación bilateral darán buen resultado en la labor para alcanzar el bienestar de los pueblos de Rusia y Corea y asegurar la paz, la estabilidad y la seguridad de la península coreana y toda la región asiática».

En este escenario, sin embargo, en donde se reafirma el histórico compromiso de Moscú con el régimen norcoreano y se busca potenciar el trabajo bilateral con Corea del Sur, la violación por parte de Pyongyang de las disposiciones del Consejo de Seguridad se transformó en un obstáculo que difícilmente podía obviarse. Para el gobierno del Presidente Medvedev el reaccionar con fuerza ante el quebrantamiento norcoreano de una disposición surgida al interior del Diálogo a Seis a Bandas y reafirmada posteriormente por Naciones Unidas implicó por primera vez en bastante tiempo emitir juicios condenatorios y absolutos a las maniobras desarrolladas por el régimen de Kim Jong II, no dejándose así lugar a justificaciones y amparos (Businessweek; 3 de junio de 2009):

"Lo que está ocurriendo es muy trágico y lamentablemente innecesario. Trabajábamos en un consolidado esfuerzo internacional para ayudar a Corea del Norte a salir de su difícil situación económica de modo que el programa nuclear que se está colocando en práctica fuese pacífico en su esencia y no cree ningún daño a los países vecinos (...) Siempre hemos tenido buenas relaciones con los líderes norcoreanos, pero lo que ha ocurrido ha generado gran alarma y preocupación (...) Necesitamos pensar en algunos medios para disuadir aquellos programas.»

La postura de la Federación de Rusia en la discusión dada al interior del Consejo de Seguridad de las Naciones Unidas, si bien insiste en enmarcar las sanciones a Corea del Norte al Artículo 41 de la Carta -no considerándose así el uso de la fuerza-, sí implica un alineamiento con la postura de los demás países del Consejo y que hace mención a que los esfuerzos de la comunidad internacional por fortalecer el Tratado de No Proliferación de Armas Nucleares se ven socavados por este tipo de acciones al margen de la legalidad internacional, poniéndose en riesgo la paz y seguridad de todo el noreste asiático.

Para el Presidente Medvedev es importante poder mantener controlada la tensión político-militar al interior de la península de Corea, pero más importante aún es lograr que su nueva diplomacia, fundada en el smart power, lleve nuevamente a Moscú a las grandes ligas de la política internacional,

industria de la defensa rusa 32 helicópteros de transporte Ka-32. (Véase: THE NATIONAL INSTITUTE FOR DEFENSE STUDIES. East Asian Strategic Review, Tokyo: The Japan Times, 2009). 
aun cuando ello signifique en determinadas situaciones apoyar decisiones que comprometan los intereses de viejos socios estratégicos, como es el caso del gobierno de Pyongyang.

\section{CONCLUSIONES}

El fin de la Administración de Vladimir Putin durante el año 2008 y la consecuente llegada al poder de Dimitri Medvedev implican de por sí la interrogante de cuánto de continuismo y cuánto de innovación se logrará apreciar en el manejo de la política exterior de Moscú en el futuro, siendo quizás arriesgado aventurar un análisis definitivo, pero pudiéndose ya identificar señales claras al respecto.

Medvedev fue un estrecho colaborador de Vladimir Putin, incluso para muchos el actual Presidente ruso ve en este a un mentor; por lo cual, esperar cambios radicales parece ilusorio, más aún considerando la gran cuota de poder e influencia que tiene Putin en su actual posición de Primer Ministro. Además, se aprecia lo anterior en la definición hecha por las autoridades moscovitas de las directrices de la política exterior, destacando el Ilamado a sus socios estratégicos a cooperar en el fortalecimiento de Naciones Unidas y de las demás instituciones internacionales en temas de seguridad.

La Federación de Rusia está empeñada en fortalecer su rol en el sistema internacional, en especial en temas que circunstancialmente podrían amenazar sus intereses nacionales; siendo en este caso demasiado preocupante una desestabilización regional producto de un escalamiento en la crisis nuclear norcoreana. Para Moscú, un colapso de la península de Corea significaría la presencia indiscutible de la República Popular China y de Estados Unidos en una zona estratégicamente relevante para sus aspiraciones, en especial para sus proyectos de conectividad y cooperación energética.

En este sentido, la Federación de Rusia ante un colapso de régimen de Pyongyang jugará sus cartas a mantener al mínimo la presencia militar internacional en la zona, a la desnuclearización de la península y a fomentar, si la reunificación se produjese, una economía dinámica que permita el desarrollo y la explotación de la región. Además, sigue siendo un desafío la concreción de la unión del ferrocarril trans-siberiano con el ferrocarril que une en este momento Norcorea con Corea del Sur, lo cual comunicaría por tierra el noreste asiático con Europa y transformaría así a la Federación de Rusia en un eje estratégico.

Desde el punto de vista de una movilización militar, todo apunta a que Moscú sería partidario del envío por parte de Naciones Unidas de fuerzas combinadas, apostando con esto el lograr desarrollar una estabilidad en la península en pro de prevenir el predominio en ella de otros poderes regionales como la República Popular China y Japón, asegurando así el desarrollo económico ruso en el largo plazo. 
En definitiva, las implicancias de un descontrol de la crisis nuclear norcoreana traería consigo una amenaza más que directa al estilo de diplomacia impulsado por Vladimir Putin y continuado por el Presidente Dmitri Medvedev y que buscó precisamente instalar a la Federación de Rusia en las «ligas mayores» de la discusión política internacional, haciendo uso de herramientas que en el pasado no eran características de ella, como son la utilización de oportunas instancias multilaterales y el smart power.

La Federación de Rusia busca recuperar el camino perdido en la escena internacional, pero el desafío está en saber jugar en un esquema que lo percibe como un actor de segundo orden en comparación con los demás gigantes regionales: Estados Unidos, Japón, la República Popular China y Corea del Sur. Vladimir Putin asumió esta realidad y se atrevió a dar un giro en la conducción de la política exterior, marcando los resultados de esta decisión el éxito o fracaso internacional de su discípulo Medvedev.

\section{BIBLIOGRAFÍA}

- BAZHANOV, E. (2003). Korea in Russia's Post Cold War Regional Political Context, Seminar on a Russian perspective concerning the confrontation on the Korean peninsula, Monterey Institute of International Studies on December 4.

- $\quad$ BLACKWILL, R.; BRAITHWAITE, R. y TANAKA, A. (1995). El compromiso de Rusia, Madrid: Instituto de Cuestiones Internacionales y Política Exterior.

- BUSZYNSKI, L. (2000). «Russia and Northeast Asia: aspirations and reality», The Pacific Review, Vol. 13, n 3. pp 399-420.

- $\quad$ EAST ASIA NONPROLIFERATION PROGRAM (EANP) (2006). «North Korean Ballistic Missile Capabilities», en: Special Report Center for Nonproliferation Studies, Monterey Institute of International Studies.

- ELLISON, H. J. (2001) «Russia, Korea and the East Asia» en Eberstadt, N. and Ellings, R. J. (Editors). Korea's Future and the Greats Powers, Seattle, University of Washington Press. pp. 164-190.

- GEORGE, A. y KEOHANE, R. O. (1991). «El concepto de interés nacional: usos y limitaciones». En: GEORGE, A. La decisión presidencial en política exterior: el uso eficaz de la información y el asesoramiento, Buenos Aires: GEL.

- IKJOONG, Y. (2004). "The development of Russian- South Korean relations under Yeltsin: In search of partnership relations base don treaties?» International Journal of Korean Unification Studies, Vol. 13, N². pp. 121-152. 
- INTERNACIONAL CRISIS GROUP (2007). "North Korea- Russia Relations: A Strained Friendship», en: Asia Briefing, $N^{\circ} 71$, Seoul/Brussels.

- IVASHENTSOV, G. (2006). "Russia-South Korea: Toward Multifaceted Partnership», International Affairs: A Russian Journal of World Politics, Diplomacy \& International Relations, Vol. 52, Issue 1. pp. 114-120.

- JOO, S. (1996). "Russian policy on Korean Unification in the Post-Cold War Era», Pacific Affairs, Spring, 69, 1. pp. 32-48

- JOO, S. (2003). «Russia and the Korean Peace Process». En: KWAK, T. y JOO, S. (editors). The Korean Peace Process and the Four Powers, Hampshire: Ashgate Publishing Company. pp. 141-167

- JOO, S. (2004). Putin's Russia and the six-party talks on North Korea, Paper prepared for presentation at the 45th Annual Convention of the International Studies Association, Montreal, Canada, March 16-20.

- $\quad$ KIM, S. (2007). North Korean Foreign Relations in the Post-cold War World, Carlisle, PA: Strategic Studies Institute.

- $\quad$ KIMURA, H. (2005). Putin's policy towards the Korean peninsula: why has Russia been losing its influence?, International Conference «Comparing Different Approaches to Conflict Prevention and Management: Korean Peninsula and the Taiwan Strait» to be held by Silk Road Studies Program, Central Asia-Caucasus Institute, Johns Hopkins University and Uppsala University on December 16 - 17.

- KO, J. y CHOI, S.: (2002). "Russia's South Korean Policy: A Comparison of Soviet and PostSoviet Dynamics», East Asian Review, Vol. 14, n² 2, Summer, South Korea, Institute for East Asian Studies. pp. 39-60.

- LEE, G. (2003). «The Political Philosophy of Juche», Stanford Journal of Asian Affairs, Volume 3, Number 1, Spring. pp. 105-112.

- MENA, C. (1989). Toma de decisiones y políticas: algunas aplicaciones a la política exterior, Buenos Aires: Programa RIAL-GEL.

- MORGuenthau, H. J. (1986). Política entre Naciones. La lucha por el poder y la paz, Buenos Aires: GEL. 
- PALACIOS, J. M. y ARANA, P. (2002). «Doctrina Militar Rusa: herencia soviética, realidades postsoviéticas, perspectiva europea», Revista CIDOB d'Afers Internacionals, N 59. pp. 81103.

- SANCHEZ, W. and CARREÑO, E. (2009). Moscow, Beijing and Latin America: Testing smart power diplomacy, Paper presented at the XXI World Congress International Political Science Association, Santiago of Chile, July 12-16.

- TAKEDA, Y. (2006). «Putin's foreign policy toward North Korea», International Relations of the Asia-Pacific, Volume 6. pp. 189-207

- TOMASSINI, L. (1987). «Elementos para el análisis de la política exterior», Estudios Internacionales, $\mathrm{N}^{\circ}$ 78. pp. 125-157.

- TROITSKIY, M. (2006). Globalization vs. National Security: External and Domestic Factors in Russian Foreign Policymaking, Paper presented at the 2006 Annual Convention of International Studies Association, San Diego, USA, March 22-25.

- VILLANUEVA, J. (2006). «Programa nuclear de Corea del Norte: una política de poder sin poder», en: Centro Argentino de Estudios Internacionales Working Paper, $N^{\circ} 14$, Buenos Aires.

- WILSON, E. J. (2008). "Hard Power, Soft Power, Smart Power», The ANNALS of the American Academy of Political and Social Science, Vol. 616, $\mathrm{N}^{\circ}$ 1. pp. 110-124

- WOO, P. (2005). «The Studies on North Korea- Russia Relations: Accomplishments and Remaining Task», East Asian Review, vol. 17, $\mathrm{n}^{\circ}$ 3, Fall, South Korea, Institute for East Asian Studies. pp. 25-51. 